\title{
The local Galactic magnetic field in the direction of Geminga (Research Note)
}

\begin{abstract}
M. Salvati
INAF - Osservatorio Astrofisico di Arcetri Largo Enrico Fermi 5, 50125 Firenze, Italy

e-mail: salvati@arcetri.astro.it

Received 5 October 2009 / Accepted 25 January 2010

ABSTRACT

Context. The Milagro hot spot A, close to the Galactic anticenter direction, has been tentatively attributed to cosmic rays from a local reservoir (at a distance $\approx 100 \mathrm{pc}$ ), freely streaming along diverging and smooth magnetic field lines. This is at variance with the geometry of the $\approx \mathrm{kpc}$ scale Galactic magnetic field, which is known to be aligned with the spiral arms.

Aims. We investigate the information available on the geometry of the magnetic field on the scales $(\approx 100 \mathrm{pc})$ of relevance here.

Methods. The magnetic field immediately upstream of the heliosphere has been investigated by previous authors by modeling the interaction of this field with the solar wind. At larger distances, we use the dispersion measure and the rotation measure of nearby pulsars (especially towards the third Galactic quadrant). Additional information about the local field towards the North Polar Spur is taken from previous studies of the diffuse radio emission and the polarization of starlight.

Results. The asymmetry of the heliosphere with respect to the incoming interstellar medium implies a magnetic field almost orthogonal to the local spiral arm, in the general direction of hot spot A, but more to the south. This is in good agreement with the nearby pulsar data on the one side, and the North Polar Spur data on the other.

Conclusions. The local magnetic field on scales of $\approx 100$ parsecs around the Sun seems to be oriented so as to provide a direct connection between the Solar System and a possible site of the Geminga supernova; the residual angular difference and the shape and orientation of the Milagro hot spot can be attributed to the field trailing in the wake of the heliosphere.
\end{abstract}

Key words. cosmic rays - supernovae: general - supernovae: individual: Geminga - ISM: magnetic fields

\section{Introduction}

The detection by Milagro of anistropies on small angular scales in the arrival directions of multi- $\mathrm{TeV}$ cosmic ray protons (dubbed hot spots A and B; Abdo et al. 2008) has stirred lively debate. There was little surprise on the detection of anisotropies at the measured level, but the expectation had been that these anisotropies would appear on larger angular scales, in agreement with the diffusion mode that accounts successfully for the propagation of cosmic rays. A positive excess in the general direction of hot spot A ("tail-in" anisotropy) had already been detected by other experiments (Tibet Air Shower Array, Amenomori et al. 2006, and Super Kamiokande I, Guillian et al. 2007), although the narrowness of the feature (only a few degrees) had not been noticed before.

Salvati \& Sacco (2008, hereafter SS) pointed out that hot spot A is in the general direction of Geminga, and suggested that a plausible source could be the Geminga supernova remnant (SNR) rather than the pulsar. The SNR would have dispersed by now and survive as only an expanding cloud of cosmic rays. Its distance could be much smaller than the present distance to the pulsar, if a positive radial velocity is assumed for the latter. This distance could be crossed by diffusion in the time elapsed since the explosion (at least for crude assumptions about the diffusion coefficient). The energetics were also found to be correct and the energy dependence of the diffusion coefficient would account for the hard spectrum $(\Gamma \sim 1.45)$ of the excess cosmic rays. Drury \& Aharonian (2008, hereafter DA) criticized SS on the grounds that the assumed diffusion coefficient was very implausible and a fully diffusive approach could not account for the narrow angular size of the hot spots. They suggested instead that some nearby "cosmic ray reservoir" was connected to the Solar System by a "magnetic funnel": the cosmic rays could then stream freely along the (diverging and smooth) field lines, while at the same time their pitch angle distribution would reduce to the observed value. A hybrid scenario was finally proposed by SS: there the "cosmic ray reservoir" coincides with the Geminga SNR; the cosmic rays have to diffuse until they reach the "first useful magnetic line", which drives them to the funnel and then to the Solar System. The initial diffusion accounts for the spectral filtering, the final streaming accounts for the angular distribution.

However, there is a major caveat. The available information about the geometry of the Galactic magnetic field (e.g., Han et al. 2006) indicates that on scales $\approx \mathrm{kpc}$ the ordered magnetic field is in the direction of the local spiral arm, and the chaotic component of the field is somewhat larger than the ordered one. In contrast, the magnetic funnel scenario requires that (on smaller scales $\approx 100 \mathrm{pc}$ ) the field is predominantly ordered, and directed toward the anticenter. In the following, we discuss evidence that this could indeed be the case.

\section{The local and very local magnetic field}

Information about the magnitude and direction of the magnetic field immediately upstream of the heliosphere (i.e., in the very local interstellar medium still unperturbed by the bow shock) can be gained by modeling the anistropies observed in several heliopause tracers (see, for instance, Ratkiewicz et al. 2008, and references therein). One obtains a very local magnetic field of 


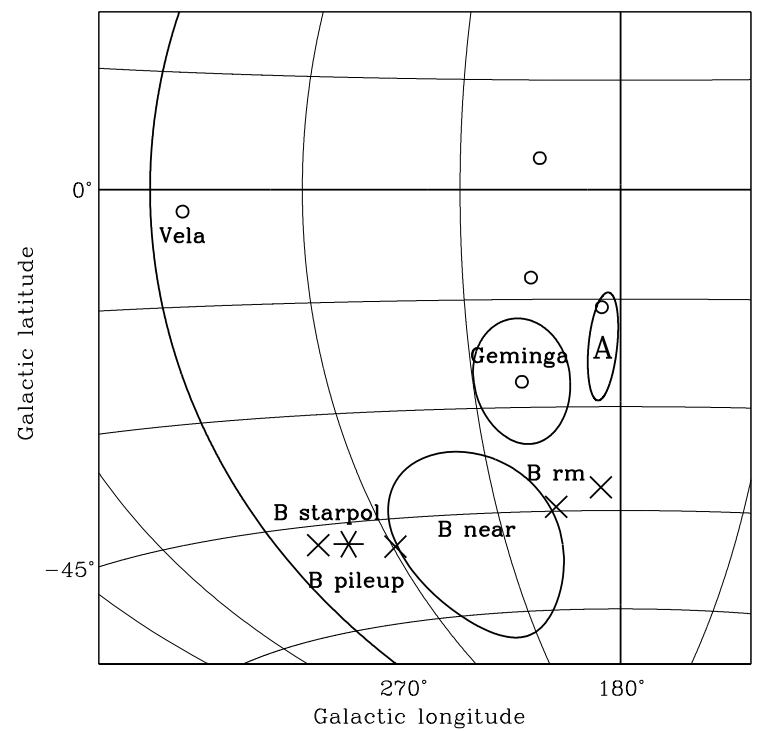

Fig. 1. Aitoff equal area projection in Galactic coordinates of the southern half of the third Galactic quadrant. See text for the meaning of the symbols. In all cases, the $B$ field is directed out of the page towards the reader.

Table 1. Nearby pulsars used in the analysis.

\begin{tabular}{cccccc}
\hline \hline Name & $\begin{array}{c}\ell_{\mathrm{II}} \\
\text { degrees }\end{array}$ & $\begin{array}{c}b_{\text {II }} \\
\text { degrees }\end{array}$ & $\begin{array}{c}\text { Dist } \\
\text { pc }\end{array}$ & $\begin{array}{c}\text { DM } \\
\mathrm{cm}^{-3} \text { pc }\end{array}$ & $\begin{array}{c}\text { RM } \\
\mathrm{rad} \mathrm{m}^{-2}\end{array}$ \\
\hline J2144-3933 & 2.8 & -49.5 & 180 & 3.35 & -2 \\
J2124-3358 & 10.9 & -45.4 & 250 & 4.60 & 1.2 \\
J0108-1431 & 140.9 & -76.8 & 130 & 2.4 & -0.3 \\
B0656+14 & 201.1 & 8.3 & 290 & 14.0 & 23.5 \\
B0950+08 & 228.9 & 43.7 & 260 & 2.96 & -0.66 \\
J0437-4715 & 253.4 & -42.0 & 160 & 2.64 & 1.5 \\
B0833-45 & 263.6 & -2.8 & 290 & 68.0 & 31.4 \\
\hline
\end{tabular}

$\sim 1.8 \mu \mathrm{G}$, oriented within the interval $203^{\circ}<\ell<231^{\circ},-58^{\circ}<$ $b<-41^{\circ}$. We note that this analysis is insensitive to the sign of the field, so that an equally admissible solution is $23^{\circ}<\ell<51^{\circ}$, $+41^{\circ}<b<+58^{\circ}$. The latter solution is plotted in Fig. 1 as a circle labeled " $B$ near".

To explore the field on scales of a few hundreds of parsecs from the Solar System, we use the dispersion measure (DM) and the rotation measure (RM) of nearby radio pulsars (Han et al. 2006). We retrieve from the ATNF Pulsar Database (Manchester et al. 2005 ${ }^{1}$ ) all pulsars with measured DM and RM, and distances less than 300 parsecs. There are seven of these objects, listed in order of increasing Galactic longitude in Table 1. Their distances are obtained from either the annual parallax or (for J0108 and J2144) the DM and an assumed model of the electron distribution. Given the relatively small volume, we approximate the magnetic field as a constant vector fully described by three independent components, which we find by minimizing the $\chi^{2}$ between the observed and the predicted $\mathrm{RM}^{2}$

$\mathrm{RM}_{\text {pred }}=0.81 \mathrm{DM}_{\mathrm{obs}}\left(\boldsymbol{B}_{\mu \mathrm{G}} \cdot \boldsymbol{n}\right)$.

On the other hand, the RM is well known to vary widely even for small angular displacements, so that the ordered component of the field is found by averaging the data over large regions of the sky. Given the small number of entries, we do not perform

\footnotetext{
${ }^{1}$ http://www.atnf.csiro.au/research/pulsar/psrcat/

${ }^{2}$ Even if each pulsar were to give only the $B$ component along the line of sight $(\boldsymbol{B} \cdot \boldsymbol{n})$, three or more pulsars widely spaced over the sky would be sufficient to constrain $B$ independent of its direction.
}

Table 2. Magnetic field obtained from various pulsar samples.

\begin{tabular}{cccccc}
\hline \hline sample & no. objects & $\begin{array}{c}\ell_{\text {II }} \\
\text { degrees }\end{array}$ & $\begin{array}{c}b_{\text {II }} \\
\text { degrees }\end{array}$ & $\begin{array}{c}\mathrm{B} \\
\mu \mathrm{G}\end{array}$ & $\chi_{\text {rid }}^{2}$ \\
\hline$<300 \mathrm{pc}$ & 7 & 16 & 45 & 1.9 & 470 \\
III quad & 4 & 5 & 42 & 3.3 & 40 \\
$<500 \mathrm{pc}$ & 18 & 6 & 28 & 2.7 & 1500 \\
III quad & 8 & 9 & 43 & 2.5 & 340 \\
$0.5-2 \mathrm{kpc}$ & 103 & 80 & -10 & 2.5 & 10000 \\
arm excl. & 57 & 100 & 6 & 3.1 & 2200 \\
\hline
\end{tabular}

any average; however, we must expect to obtain a $\chi^{2}$ very much higher than $\approx 1$ per degree of freedom. We use this estimator only to draw some qualitative guesses. The values of DM and RM for two particular objects (B0656+14 and B0833-45) are by far larger than the other values, as one would expect because of their location in the Monogem and Vela SNR, respectively. This adds another caveat to our results, since a dense, young SNR could be dominated by a local magnetic field of its own.

When we retain all seven pulsars in the fit, we obtain $B \sim$ $1.9 \mu \mathrm{G}, \ell \sim 16^{\circ}$, and $b \sim 45^{\circ}$ with a reduced $\chi^{2}$ of around 470 (!). When instead we retain only the four pulsars lying in the third Galactic quadrant, since the excess cosmic rays reach the Solar System from this general direction, we obtain $B \sim 3.3 \mu \mathrm{G}$, $\ell \sim 5^{\circ}$, and $b \sim 42^{\circ}$ with a reduced $\chi^{2}$ of only (!) 40. As a check of our findings, we repeated the analysis for all pulsars with distances smaller than 500 parsecs: this step, on the one hand, made the fit sensitive to fields on scales somewhat larger than those of interest, and, on the other, improved the statistics by increasing the sample to 18 objects in total, and to 8 in the third Galactic quadrant. Finally, we fitted all pulsars with distances between 500 and 2000 parsecs (103 objects), which should reproduce the azimuthal geometry already established by previous authors. On these large scales, we include a portion of the Sagittarius - Carina arm, where the field is known to reverse direction (Han et al. 2006); to prevent our sample being affected by the arm, we therefore fitted a subsample that included only pulsars lying outside the arm (57 objects).

Our results are summarized in Table 2. One sees that the $<500$ pc sample provides results in broad agreement with the $<300$ pc sample, while the $0.5-2 \mathrm{kpc}$ sample clearly indicates a rotation of the field, which becomes (more or less) aligned with the Galactic plane in the direction of the local spiral arm. We note in particular that restricting our analysis to the third Galactic quadrant does not appreciably change the field, but makes the reduced $\chi^{2}$ substantially smaller. The reduced $\chi^{2}$ also becomes substantially smaller in the large-scale sample, as expected, if one excludes the pulsars inside the Sagittarius-Carina arm.

We regard the substantial agreement between the first four sets of values in Table 2 as an indication that our procedure is meaningful. Furthermore, the substantial agreement between the pulsar-derived magnetic field (on scales $\approx 100 \mathrm{pc}$ ) and the very local, heliopause-derived magnetic field is a hint that in our Galactic neighborhood the magnetic field is relatively smooth. An additional hint of the field smoothness (a prerequisite for the validity of the funnel scenario) is the significant decrease in the reduced $\chi^{2}$ in the third quadrant relative to the all-sky value. The two $<300$ pc pulsar-derived solutions are plotted as two crosses labeled " $B$ rm" in Fig. 1. We do not compute a confidence region for the $B$ field direction from the $\chi^{2}$ distribution because of the mentioned caveats; however, an order-of-magnitude estimate of the errors can be derived from the difference between the two solutions. 
The structure of the magnetic field toward the Galactic center is loosely constrained by the pulsar data, which are consistent only with a geometry more complex than a uniform field. A clearer picture can be obtained by modeling the intensity and polarization of the nearby extended radio emission (Wolleben 2007) and the polarization of the light from nearby stars (Frisch 2009).

In this general direction, the interstellar medium has been perturbed by a series of explosions probably from stars in the Sco-Cen association. The radio intensity and radio polarization maps show traces of several shells, the most prominent of which is the North Polar Spur. One of the shells, called "Shell 1" by Wolleben (2007), may have reached the Sun. To account for both the radio and the optical data, the magnetic field in the perturbed region is described as a uniform field outside the shells and, within the shell thickness, as a compressed field lying along the meridian circles. The radio data require two different shells, while the optical data can be fitted with Shell 1 only, and help us to constrain its parameters within the large radio-derived confidence region.

The star symbol labeled " $B$ starpol" in Fig. 1 is the direction of the uniform field inside which Shell 1 is expanding (Frisch 2009, no errors given). This would be the direction of the field outside the heliosphere if Shell 1 had not yet reached us. Otherwise, the field would be that compressed along the local meridian line of the shell: the two crosses labeled " $B$ pileup" represent two possible choices of the shell center. We note the near coincidence of " $B$ starpol" and " $B$ pileup", which is caused by the shell expansion center being offset by almost $90^{\circ}$ with respect to " $B$ starpol".

Figure 1 summarizes our findings. Here the southern half of the third Galactic quadrant is plotted in an Aitoff equal area projection. The various estimates of the $B$ field direction have already been discussed. For the sake of comparison, all are represented as the respective points at $-\infty$, but they pertain to different physical regions: $B_{\mathrm{rm}}$ should be valid at $\approx 100 \mathrm{pc}$ in the third quadrant, $B_{\text {near }}$ and $B_{\text {pileup }}$ should be valid only very close to the Sun, and $B_{\text {starpol }}$ should be valid at $\approx 100 \mathrm{pc}$ in the first quadrant. In this region, the field has been heavily distorted by the expansion of the radio shells; however, we plot here the unperturbed, pre-shell field, so that we can draw meaningful conclusions from its smooth connection with $B_{\text {near }}$ and $B_{\text {rm }}$ (see Sect. 3 and Figs. 2 and 3 ).

The hot spot $\mathrm{A}$ and the heliotail direction are represented by the ellipse labeled " $A$ " and the small dot inside it. Finally, the three dots along $\ell \approx 200^{\circ}$ are, from top to bottom: the present position of the Geminga pulsar; the position it would have had on explosion if its motion were parallel to the plane of the sky with the measured proper motion value; and the position it would have had if the explosion had occurred at the "minimum" distance of $65 \mathrm{pc}$, i.e., with a positive $160 \mathrm{~km} \mathrm{~s}^{-1}$ radial velocity included (see SS). In the latter two cases, the time elapsed since the explosion is assumed to equal the spindown age of the pulsar, $3.4 \times 10^{5}$ yr (Bignami \& Caraveo 1996). Around the third dot, we have drawn a circle of $10 \mathrm{pc}$ radius, representing a fully developed SNR.

\section{Discussion and conclusions}

We first emphasize the geometry displayed in Fig. 1: the direction of the local magnetic field, the direction of hot spot A, and the direction to a possible location for the Geminga SNR all lie

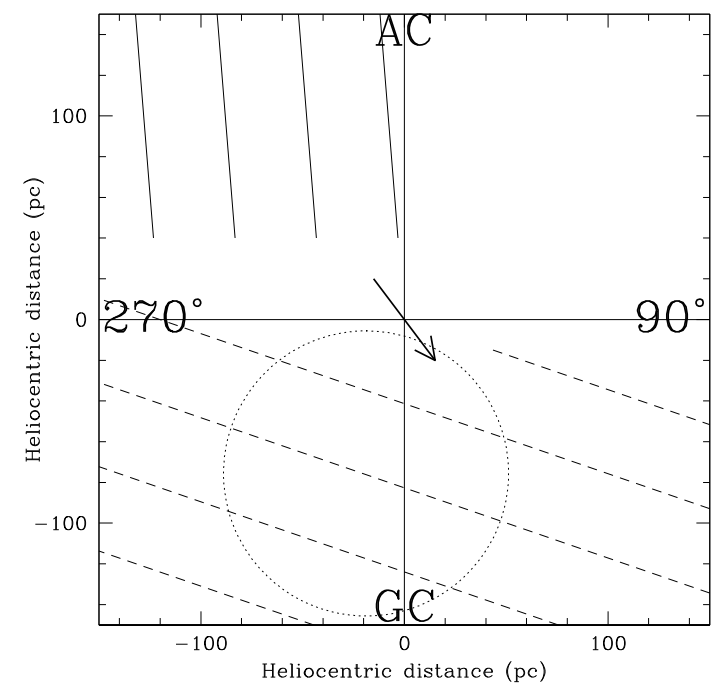

Fig. 2. Orthogonal projection onto the Galactic plane of the pulsarderived field (the rightmost cross of Fig. 1, solid lines), the unperturbed radio-optical derived field (the star symbol of Fig. 1, dashed lines), and the radio Shell 1 (based on the assumption that it has not reached the Sun yet, dotted circle). The heavy line through the center is the heliospheric derived, very local field. Its arrow indicates the field orientation. The axes are labeled GC (Galactic center), AC (anticenter), 90 ${ }^{\circ}$, and $270^{\circ}$ (for the Galactic longitude).

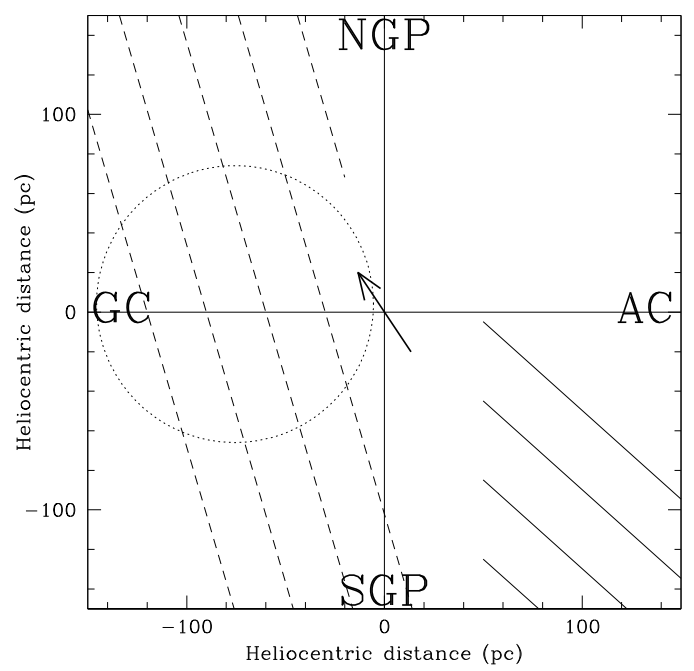

Fig. 3. The same as Fig. 2, for the meridian plane $\ell=180^{\circ}$. The vertical axis is labeled NGP (North Galactic pole) and SGP (South Galactic pole).

within a few degrees from each other ${ }^{3}$. Apart from the hot spot, all the other directions in Fig. 1 are not directly measured, and are obtained by modeling the available datasets, which are not always plentiful. Nonetheless, if we were to interpret these results at face value, one of the main objections to a diffusionplus-funnel scenario could be removed: the field on the relevant scales seems to be almost orthogonal to the large-scale one, and to point in the correct direction.

\footnotetext{
3 Battaner et al. (2009) developed a model for the dipole-like Milagro anisotropy, which is different from the point-like anisotropy discussed here. Their model succeeds in accounting for the dipole based on the assumption of a local magnetic field basically aligned with the local spiral arm, i.e., at a large angle to the one derived here. But if one assumes a streaming motion of the cosmic rays along the magnetic field, in addition to the orthogonal motion derived by them based on an adhoc turbulent stress, the two estimates can be reconciled.
} 
The second result concerns the smoothness of the local field, which is necessary if the cosmic rays are to stream freely along the magnetic funnel and be focused within a narrow range of pitch angles. Evidence of this smoothness (admittedly meager) comes from two findings. One is the dramatic decline in the reduced $\chi^{2}$ when one selects for modeling only the pulsars lying in the third Galactic quadrant. The other is the near coincidence between the directions of the very local, heliopause-derived magnetic field (" $B$ near"), and the $\approx 100$ pc scale one, either pulsarderived (" $B \mathrm{rm}$ "), or radio-optical derived (for the unperturbed configuration, " $B$ starpol"). One notes that there is a regular and smooth "rotation" of the $B$ field direction, which points approximately toward the Galactic center when the field is determined in the third Galactic quadrant, grows in Galactic longitude by about $30^{\circ}$ on approaching the Solar System, and increases by an additional $30^{\circ}$ when the (unperturbed) field is determined in the direction of the Galactic center.

The envisaged geometry we sketch in Figs. 2 and 3 includes the projection onto the Galactic plane and the meridian plane $\ell=180^{\circ}$, respectively, of the local and very local magnetic field, and the outline of Shell 1 of Wolleben (2007). The crudeness of the sketch appears to show a sharp bend at the solar position, but an equally valid (and equally arbitrary) representation could involve magnetic lines with a curvature radius as large as the figures themselves. In addition, the true rotation in three dimensions is equal to only 46 degrees, which is strongly amplified by projection effects. Finally, the dashed lines inside the shell indicate the pre-shell magnetic lines: after the shell has overtaken them, these lines are draped along the shell surface.

We do not regard as a major discrepancy the residual angular separation between the assumed directions of the Geminga SNR, " $B$ near", " $B \mathrm{rm}$ ", and the true position of hot spot A. However, some arguments can be considered that account for the discrepancy.

As argued in SS, the SNR responsible for the cosmic ray reservoir (Geminga or other) should be close to the magnetic funnel, so that diffusion with reasonable coefficients could account for the propagation of the cosmic rays from the SNR to the funnel in the time elapsed since the explosion. At the same time, however, the SNR should not lie directly on the "first useful field line", otherwise one would miss the energy filtering, which is needed to explain the spectral hardness of the cosmic ray excess.

Secondly, a small meandering of the magnetic field, sufficient to account for the angular difference between " $B \mathrm{rm}$ " and " $B$ near", is not only plausible, but indeed very likely. The important point is that these small deflections over several tens of parsecs are by far insufficient to affect the free streaming of the cosmic rays.

Thirdly, the true position of hot spot $\mathrm{A}$ is perhaps determined by the direction of the very local magnetic field in the wake of the heliosphere. The direction of " $B$ near" depicted in Fig. 1 refers to the field ahead of the heliosphere, before any interaction with it (Ratkiewicz et al. 2008). After the wind, the field should become more aligned with the heliotail, and it is plausible than the alignment lasts for several times the distance to the heliopause, i.e., for about $\lesssim 10^{16} \mathrm{~cm}$. This distance is comparable to the Larmor radius of a $10-\mathrm{TeV}$ particle; the radius of curvature needed for a $20^{\circ}$ swing over this distance is of course larger still, so the free streaming of the cosmic rays should not be disrupted.

We note that the ambient magnetic field lines will tend to wrap around the heliosphere in the plane passing through the apex that contains the field and wind directions, while they will tend to slip apart on the two sides; the cosmic rays will then be focused in the said plane, and defocused on the two sides. This corresponds roughly to the elliptical shape and the position angle of hot spot A. Qualitatively, the pile-up of the lines toward the heliotail could also account for the gradient observed in hot spot A along the major axis, with the maximum on the heliotail side.

The geometry of the magnetic field that we have discussed thus far perhaps cannot be tightly constrained by the available evidence. In all cases, it is unable to provide a quantitative estimate of the anisotropy amplitude. To achieve this, one should follow with high spatial and temporal resolution the expansion of the cosmic ray cloud injected by the supernova, including the individual field irregularities throughout the cloud volume. The cloud, which we assume to be spherical, may be elongated in one dimension, or have a complicated topology. The best we can do at the moment is to show that the observed anisotropy can be sustained by a minuscule gradient in the density of the cosmic rays, a gradient not implausible for a location relatively close to a relatively recent supernova explosion.

The energy flux measured from hot spot A (Abdo et al. 2008) is

$\Phi \sim 5 \times 10^{-4} \times 6.7 \times 10^{-6} \sim 3.3 \times 10^{-9} \mathrm{erg} \mathrm{cm}^{-2} \mathrm{~s}^{-1} \mathrm{sr}^{-1}$.

The magnetic funnel on the injection side is about 20 times narrower than on the Sun side (see DA), and the square of the particle pitch angle scales inversely by the same factor, so that $\Phi$ is constant. Hence the required density is

$n \sim 4 \pi \frac{\Phi}{\mathrm{c}} \sim 1.4 \times 10^{-18} \mathrm{erg} \mathrm{cm}^{-3}$.

If the supernova explosion injects $10^{50} \mathrm{erg}$ in cosmic rays with the same spectrum as the general cosmic ray population, the 10$\mathrm{TeV}$ reservoir amounts to $1.7 \times 10^{47} \mathrm{erg}$. Spreading this reservoir across a sphere of radius $100 \mathrm{pc}$ (the length of the funnel suggested by DA, and a plausible distance for the Geminga explosion), one obains $n \sim 1.4 \times 10^{-15} \mathrm{erg} \mathrm{cm}^{-3}$, which is three orders of magnitude greater than Eq. (2).

Alternatively, we can compute the cloud volume corresponding to the density of Eq. (2), $V \sim 1.2 \times 10^{65} \mathrm{~cm}^{3}$, and deduce a diffusion coefficient. Setting the time $t$ since the explosion of Geminga to equal $3.4 \times 10^{5} \mathrm{yr}$ (Bignami \& Caraveo 1996), we find that

$D=\left(\frac{3 V}{4 \pi}\right)^{2 / 3} \times \frac{1}{4 t} \sim 2.2 \times 10^{29} \mathrm{~cm}^{2} \mathrm{~s}^{-1}$.

The above value of $D$ is not far from what is usually assumed in cosmic ray modeling (e.g., Hooper et al. 2009), and is another plausibility argument in favor of our suggestion that hot spot A could be the first example of direct cosmic ray astronomy.

\section{References}

Abdo, A. A., Allen, B., Aune, T., et al. 2008, Phys. Rev. Lett., 101, 221101 Amenomori, M., Ayabe, S., Bi, X. J., et al. 2006, Science, 314, 439 Battaner, E., Castellano, J., \& Masip, M. 2009, ApJ, 703, L90 Bignami, G. F., \& Caraveo, P. A. 1996, ARA\&A, 34, 331 Drury, L. O.'C., \& Aharonian, F. A. 2008, Aph, 29, 420 (DA) Frisch, P. C. 2009, Space Sci. Rev., 143, 191

Guillian, G., Hosaka, J., Ishihara, K., et al. 2007, Phys. Rev. D, 75, 062003 Han, J. L., Manchester, R. N., Lyne, A. G., Quiao, G. J., \& van Straten, W. 2006, ApJ, 642, 868

Hooper, D., Blasi, P., \& Serpico, P. D. 2009, JCAP, 1, 25

Manchester, R. N., Hobbs, G. B., Teoh, A., \& Hobbs, M. 2005, AJ, 129, 1993

Ratkiewicz, R., Ben-Jaffel, L., \& Grygorczuk, J. 2008, in Numerical Modeling of Space Plasma Flows, ed. N. V. Pogorelov, E. Audit, \& G. P. Zank, ASP Conf. Ser., 385, 189

Salvati, M., \& Sacco, B. 2008, A\&A, 485, 527 (SS)

Wolleben, M. 2007, ApJ, 664, 349 